\title{
Cirurgia filtrante não penetrante: conceito, técnicas e resultados
}

\author{
Non-penetrating filtering surgery:concept, technique and results
}

\author{
Ricardo Augusto Paletta Guedes ${ }^{1}$ \\ Vanessa Maria Paletta Guedes ${ }^{2}$
}

\begin{tabular}{|l|}
\hline RESUMO \\
\hline A cirurgia filtrante não penetrante tem sido alvo de muita discussão nos \\
últimos anos. As duas técnicas mais praticadas são a viscocanalostomia e \\
a esclerectomia profunda não penetrante com trabeculectomia externa. Elas \\
têm por objetivo abaixar a pressão intra-ocular, diminuindo a resistência ao \\
escoamento do humor aquoso. Os pontos em comum destas duas técnicas \\
são: a retirada de um retalho escleral profundo, pré-ciliar e pré-coroidiano; \\
abertura e retirada da parede externa do canal de Schlemm; além da retirada \\
do estroma corneano, situado àfrente do trabeculado anteriore da membrana \\
de Descemet. Ambas as técnicas levam à formação de um espaço denomi- \\
nado câmara de descompressão. O humor aquoso deixa a câmara anterior, \\
atravessando a fina membrana trabecular residual e permanece na câmara de \\
descompressão até ser absorvido por diferentes maneiras. Na viscocanalos- \\
tomia, há a injeção de um produto viscoelástico nas duas extremidades \\
abertas do canal de Schlemm, a fim de abrir a luz do canal e seus eferentes, \\
para que o humor aquoso drene mais facilmente. Já na esclerectomia \\
profunda com trabeculectomia externa, o ponto essencial é a retirada da \\
membrana trabecular externa (trabeculadojuxtacanalicular e parede interna \\
do canal de Schlemm). Os estudos retrospectivos e prospectivos de ambas \\
as técnicas, que as comparam à trabeculectomia, relatam taxas de sucesso \\
similares, com menor incidência de complicações pós-operatórias e recupe- \\
ração visual mais rápida com a cirurgia não penetrante. O objetivo deste \\
artigo é expor e descrever as diferentes técnicas, seus mecanismos de ação, \\
seus resultados através da análise da literatura. \\
\hline
\end{tabular}

Descritores: Glaucoma/cirurgia; Cirurgia filtrante; Pressão intra-ocular/fisiologia; Cuidados pós-operatórios; Acuidade visual; Humor aquoso/secreção; Trabeculectomia; Esclerostomia

\section{INTRODUÇÃO}

Trabalho realizado no Serviço de Oftalmologia do Hospital Universitário da Universidade Federal de Juiz de Fora e no Serviço de Oftalmologia do Hospital Dr. João Felício

${ }^{1}$ Médico oftalmologista do Hospital Universitário da Universidade Federal de Juiz de Fora - UFJF - Juiz de Fora (MG) - Brasil, e do Hospital Dr. João Felício - Juiz de Fora (MG) - Brasil.

Médica oftalmologista do Hospital Dr. João Felício Juiz de Fora (MG) - Brasil.

Endereço para correspondência: Ricardo Augusto Paletta Guedes - Rua Oscar Vidal, 79 - Juiz de Fora (MG) CEP 36010-060

E-mail: palettaguedes@yahoo.com

Recebido para publicação em 26.04.2005

Versão revisada em 14.11.2005

Aprovação em 30.11.2005

\begin{tabular}{l} 
INTRODUÇÃO \\
\hline Na ausência de tratamento neuroprotetor efetivo ou que permita a \\
regeneração do nervo óptico, o tratamento do glaucoma primário de ângulo \\
aberto (GPAA) consiste, ainda, em primeira intenção, na diminuição da \\
pressão intra-ocular (PIO) ${ }^{(1-2)}$. \\
O tratamento cirúrgico do GPAA consiste em se criar uma nova via de \\
drenagem e absorção do humor aquoso. Ele está indicado quando existe a \\
impossibilidade de se atingir uma pressão intra-ocular adequada (PIO-alvo) \\
somente com tratamento clínico hipotensor e/ou aplicação de laser ${ }^{(1-3)}$. \\
A trabeculectomia tem sido a cirurgia de escolha para o controle pressó- \\
rico do GPAA desde o final da década de 60 . Seu sucesso se deu principal- \\
mente pelo fato dela permitir uma redução pressórica equivalente à redução \\
obtida com as cirurgias de espessura total realizadas na época, porém com a
\end{tabular}


vantagem de diminuir acentuadamente os índices de complicações por utilizar uma fístula protegida por um retalho escleral $^{(1-2)}$.

A trabeculectomia consiste em se criar uma comunicação direta entre a câmara anterior e os espaços subconjuntivais, ressecando-se o trabeculado em toda sua espessura. O local da trabeculectomia é recoberto por um retalho de esclera, previamente dissecado, com a finalidade de se modular a drenagem do humor aquoso. A descompressão brusca do globo ocular, a filtração excessiva e a hipotonia, causadas pela abertura da câmara anterior, são complicações potenciais no pós-operatório desta técnica cirúrgica, podendo levar à baixa de visão importante, comprometendo o prognóstico visual do paciente ${ }^{(1-2)}$.

A eclosão de novas técnicas cirúrgicas não penetrantes visa diminuir o risco daqueles tipos de complicações ligados à abertura da câmara anterior. As cirurgias filtrantes não penetrantes têm por finalidade diminuir a pressão intra-ocular reduzindo a resistência ao escoamento do humor aquoso, que está anormalmente elevada nos pacientes glaucomatosos ${ }^{(4-7)}$. O local exato da resistência ao escoamento do humor aquoso nos pacientes portadores de GPAA ainda é motivo de discussão, porém admite-se geralmente que o trabeculado juxtacanalicular e a parede interna do canal de Schlemm respondem a $75 \%$ desta resistência ${ }^{(5)}$ e que $25 \%$ estão a cargo da parede externa do canal e a esclera adjacente ${ }^{(6)}$. O próprio colapso do canal pode também ser responsável pela hipertonia ocular ${ }^{(7)}$.

O objetivo deste artigo é expor e descrever as diferentes técnicas, seus mecanismos de ação, seus resultados através da análise da literatura.

\section{REVISÃO BIBLIOGRÁFICA}

\section{1 - Histórico}

O precursor da cirurgia filtrante não penetrante é sem dúvida o cirurgião russo Krasnov, que propõe em 1964 uma intervenção que ele batiza sinusotomia. A sinusotomia consistia em ressecar uma lamela escleral acima do canal de Schlemm e exteriorizar o canal em $120^{\circ(8)}$. A ausência de publicação de resultados convincentes em longo prazo levou ao abandono desta técnica.

Nos anos 70, o professor Fyodorov, aproveitando a idéia de Krasnov de uma resistência intraescleral ao escoamento, propõe uma outra intervenção: a esclerectomia profunda não penetrante. Ela consiste na abertura do canal de Schlemm (sinusotomia) pela via posterior, associada à excisão de um retalho escleral profundo pré-ciliar. Neste ponto, duas escolas se divergem: uma que preconiza abertura do canal e em seguida a excisão da parede interna do canal juntamente com o trabeculado cribriforme ou juxta-canalicular (Zimmermann et al., ${ }^{(9-10)}$ ); outra que propõe a abertura do canal seguida de uma dissecção profunda até a córnea na procura de uma filtração através da

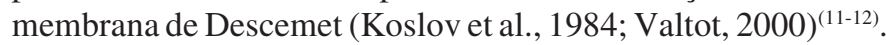
No início dos anos 90, Stegmann et al., começou a praticar a esclerectomia profunda não penetrante (EPNP) à moda russa.
Pouco a pouco, ele introduziu algumas modificações: uma delaminação da membrana de Descemet mais anterior e a injeção de ácido hialurônico de alta viscosidade no interior do canal de Schlemm, através de uma microcânula especial, após a retirada do bloco córneo-escleral. Ele ainda preenche o espaço situado entre o plano profundo e o retalho escleral superficial com o ácido hialurônico, suturando de maneira apertada este retalho. O ácido hialurônico é reabsorvido em alguns dias. Stegmann não observa, praticamente nunca, bolha filtrante ${ }^{(13)}$.

Entre 1990 e 1995, o colombiano Arenas-Archilla modifica progressivamente a sinusotomia de Krasnov. Ele pratica a sinusotomia retirando toda a esclera acima do canal de Schlemm, assim como sua parede externa, sem retalho escleral superficial. Ele denomina esta técnica de trabeculectomia ab externo. Para obter uma melhor drenagem do humor aquoso, ele promove uma raspagem do canal remanescente com uma lâmina de diamante. Com isto, ele provoca um afinamento do trabeculado ${ }^{(14)}$.

A esclerectomia profunda não penetrante foi introduzida na Europa ocidental por Demailly et al., em $1996^{(15)}$. Na tentativa de se melhorar a filtração do humor aquoso, além de ressecar a membrana correspondente à parede interna do canal de Schlemm e o trabeculado mais externo, Demailly, seguindo o exemplo de Koslov et al., ${ }^{(16)}$ utilizou um implante de colágeno no leito escleral profundo. Seus resultados não mostraram diferenças comparando-se o implante de colágeno com a aplicação de 5-fluoro-uracil ${ }^{(15)}$.

No Brasil, a esclerectomia profunda não penetrante teve seus primeiros resultados publicados em 2001 por Guedes e Guedes $^{(17)}$.

\section{2 - Técnicas e mecanismos de ação}

As técnicas de cirurgia filtrante não penetrante mais comumente realizadas são a viscocanalostomia e a esclerectomia profunda não penetrante com trabeculectomia externa.

\section{Viscocanalostomia}

A intervenção começa com dissecção conjuntivo-tenoniana base fórnice e em seguida é realizado um retalho escleral superficial ( 5 por $5 \mathrm{~mm}$ ), dissecado até a córnea clara. Um segundo retalho escleral triangular é dissecado profundamente em um plano pré-coroidiano, permitindo chegar, anteriormente, até o esporão escleral e, logo em seguida, o canal de Schlemm. O canal de Schlemm é aberto e a dissecção se continua mais à frente até a exposição da membrana de Descemet ${ }^{(4,13)}$.

A exérese deste retalho profundo é realizada e o espaço vazio restante é chamado de câmara de descompressão ou lago intra-escleral.

Em seguida, injeta-se um produto de alta viscoelasticidade dentro das duas extremidades abertas do canal de Schlemm a fim de dilatá-lo. O retalho escleral superficial é suturado de maneira apertada com mononylon 10.0 e o viscoelástico é injetado dentro da câmara de descompressão abaixo do retalho escleral no fim da intervenção, antes da sutura do plano conjuntivo-tenoniano com fio absorvível $8.0^{(4,13)}$. 
A injeção de uma substância viscoelástica dentro do canal de Schlemm tem por objetivo dilatar o canal e seus eferentes a fim de facilitar a drenagem do humor aquoso. O retalho escleral é suturado de maneira apertada para que o humor aquoso permaneça no lago intra-escleral e não atinja os espaços subconjuntivais, não havendo a formação de uma bolha de filtração. Teoricamente a via fisiológica de drenagem seria restaurada e se evitaria o problema de filtração subconjuntival exposta a uma fibrose, comprometendo o sucesso cirúrgico ${ }^{(4)}$.

Um estudo realizado em macacos mostrou que, além da dilatação canalicular e de seus eferentes, a injeção de viscoelástico levaria também a uma ruptura das paredes do canal e do trabeculado adjacente, de maneira que a intervenção funcionaria como uma trabeculotomia, ou seja, microperfurações trabeculares que transformariam a cirurgia em uma intervenção penetrante na câmara anterior ${ }^{(18)}$.

\section{Esclerectomia profunda não penetrante com trabeculectomia externa}

Inicialmente, realiza-se uma dissecção conjuntivo-tenoniana de base fórnice. Em seguida, passa-se à confecção do retalho escleral superficial, com $1 / 3$ da espessura total da esclera, até a córnea clara ( 5 por $5 \mathrm{~mm}$ ). O segundo retalho escleral profundo, em um plano pré-coroidiano, é realizado até a abertura do canal de Schlemm ${ }^{(19)}$.

Após a exposição do canal, faz-se a exérese do retalho profundo. Uma vez que a esclerectomia profunda foi realizada, inicia-se o procedimento da trabeculectomia externa, isto é, a ablação seletiva de parte do trabeculado responsável por grande parte da resistência ao escoamento do humor aquoso (a parede interna do canal de Schlemm e as camadas trabeculares adjacentes), ao mesmo tempo em que as camadas trabeculares internas permacem intactas, garantindo, assim, a ausência de abertura da câmara anterior ${ }^{(4,19)}$.

A exérese do trabeculado mais externo é realizada com o auxílio de uma pinça delicada sem dente, que faz a retirada de uma membrana, a qual é denominada membrana trabecular externa. Esta se cliva facilmente dos planos trabeculares mais profundos, pois existe um plano de clivagem intratrabecular ${ }^{(19)}$. Bresson-Dumont et al., descreveram um método particular para a retirada da membrana por aspiração, através de uma cânula especial, método este batizado de trabeculoaspiração ${ }^{(20)}$.

Estudos recentes demonstraram, através da análise por microscopia confocal, que esta membrana trabecular externa retirada no curso desta cirurgia possui uma espessura constante de 34,4 \pm 7,3 micra, correspondendo à parede interna do canal de Schlemm, trabeculado cribriforme e parte do trabeculado córneo-escleral ${ }^{(21)}$.

O retalho escleral superficial pode ou não ser suturado. Em caso de se optar pela sutura, faz-se um ponto frouxo com mononylon 10.0. Finaliza-se a intervenção suturando a conjuntiva com fio absorvível 8.0.

A exérese do retalho escleral profundo e do estroma corneano situado à frente do trabeculado anterior e da membrana de Descemet permite a passagem do humor aquoso da câmara anterior à câmara de descompressão. Levando-se em conta que a membrana de Descemet não é suficientemente permeável, que permita uma diminuição tensional eficaz em casos de glaucoma, é possível melhorar o escoamento do humor aquoso, após a esclerectomia profunda, através da retirada da parede interna do canal e do trabeculado subjacente ${ }^{(4)}$.

Nesta técnica, o ponto essencial é a ablação da membrana trabecular externa, pois ela contém os sítios responsáveis pela maior parte da resistência ao escoamento do humor aquoso nos pacientes glaucomatosos ${ }^{(5,7)}$.

$\mathrm{Na}$ maior parte das esclerectomias profundas com trabeculectomia externa, há a formação de bolha de filtração, o que demonstra a passagem do humor aquoso da câmara de descompressão até os espaços subconjuntivais. Esta bolha de filtração, no entanto, é bem mais modesta e difusa que a da trabeculectomia clássica ${ }^{(4)}$.

O humor aquoso represado na câmara de descompressão pode tomar três direções:

a) Espaços subconjuntivais, formando a bolha de filtração;

b) Via uveo-escleral, pelo leito da esclerectomia. Esta hipótese é confirmada pela visualização em biomicroscopia ultrasônica (UBM), em $60 \%$ dos casos, de uma zona supraciliar hipoecogênica, sugerindo a passagem do humor aquoso através do espaço supracoroidiano ${ }^{(22)}$;

c) Escoamento transescleral. A hiporrefletividade da esclera ao redor da câmara de descompressão, visualizada em $50 \%$ dos casos em UBM, demonstra um escoamento transescleral do humor aquoso ${ }^{(22)}$. Recentemente, um estudo em modelo animal demonstrou o aparecimento de vasos neoformados coletores de humor aquoso na esclera ao redor da câmara de descompressão ${ }^{(23)}$.

\section{3 - Utilização de adjuvantes}

Com a finalidade de se evitar uma proliferação fibroblástica e de se melhorar a filtração, pode-se lançar mão da utilização de implantes ou antimetabólitos ${ }^{(24)}$.

As indicações e as doses para a utilização de antimetabólitos são as mesmas usadas na trabeculectomia clássica, quais sejam: pacientes jovens (menos de 45 anos), da raça negra, após cirurgia ocular, em uso de medicação tópica há longa data $^{(1)}$. O uso de mitomicina $\mathrm{C}$ ou de 5 fluoro-uracil depende da experiência de cada cirurgião.

Os implantes são colocados no final da intervenção no leito escleral residual antes do fechamento do retalho escleral superficial. Existem três tipos principais de implantes no mercado internacional:

a) Implante de colágeno (Aquaflow ${ }^{\circledR}$ ): criado pelo russo Koslov, foi o primeiro a ser desenvolvido para esta função. É biocompatível, cilíndrico (dimensões $2,5 \times 1 \times 0,5 \mathrm{~mm}$ ), fabricado a partir de tecido escleral de porco. Ele se reabsorve em 6 a 9 meses e deve ser suturado no centro do leito escleral mais anteriormente possível com um ponto de mononylon $10.0^{(15-16)}$.

b) Implante de ácido hialurônico (SK-gel $\left.{ }^{\circledR}\right)$ : triangular equilateral com lados de 3,5 mm ou 3,5x4,5 mm, biocompatível. Ele ocupa todo o espaço da câmara de descompressão e se reabsorve em 2 meses $^{(24-25)}$. 
c) Implante de acrílico hidrofílico ( $\mathrm{T}$-flux $\left.{ }^{\circledR}\right)$ : em forma de $\mathrm{T}$, com 4 mm de largura e 2,75 mm de comprimento, não reabsovível. Os dois braços do T são introduzidos nas extremidades abertas do canal de Schlemm e o corpo e fixado na esclera com pontos de mononylon $10.0^{(24)}$.

\section{4 - Indicações}

As indicações da cirurgia não penetrante na literatura são numerosas e correspondem aos glaucomas primários ou secundários de ângulo aberto ${ }^{(24)}$. Os tipos de glaucoma que são passíveis de tratamento cirúrgico por esta técnica são os seguintes:

a) Glaucoma primário de ângulo aberto;

b) Glaucoma pseudoexfoliativo;

c) Glaucoma pigmentário;

d) Glaucoma juvenil;

e) Glaucoma inflamatório;

f) Glaucoma traumático;

g) Glaucoma do pseudofácico e do afácico;

h) Glaucoma de pressão normal.

A cirurgia não penetrante não parece interessante para os glaucomas de ângulo fechado, pois o obstáculo ao escoamento do humor aquoso se situa além da região retirada no procedimento, tornando a intervenção ineficaz. No entanto, é possível realizá-la nestes casos, praticando-se uma iridectomia periférica a laser no local correspondente ao sítio de filtração ${ }^{(24)}$.

Há um consenso, portanto, entre a maioria dos autores, que a melhor indicação para a cirurgia não penetrante é o glaucoma primário de ângulo aberto ${ }^{(24)}$.

\section{RESULTADOS}

Os resultados serão demonstrados separadamente para cada técnica.

\section{Viscocanalostomia - Estudos retrospectivos}

Carassa et al., publica os resultados da viscocanalostomia realizada em 33 olhos de 33 pacientes. Em 4 casos, o procedimento foi convertido em trabeculectomia devido a perfuração per-operatória. Aos 3 meses de acompanhamento, 86,2\% dos olhos tinham uma pressão intra-ocular abaixo de $21 \mathrm{mmHg}$ sem tratamento hipotensor e 79,3\% tinham uma pressão abaixo de $16 \mathrm{mmHg}$ sem tratamento. A média tensional passava de 27,7 \pm 9,5 $\mathrm{mmHg}$ no pré-operatório para $12,0 \pm 3,0 \mathrm{mmHg}$ no pósoperatório $^{(26)}$.

Em um estudo realizado em 214 olhos de 157 pacientes africanos, portadores de glaucoma primário de ângulo aberto, com pressão intra-ocular média pré-operatória de 47,4 \pm 13,0 $\mathrm{mmHg}$, Stegmann descreve, após 35 meses de acompanhamento, uma pressão intra-ocular média de 16,9 \$ 8,0 mmHg após a viscocanalostomia. A taxa de sucesso para uma PIO menor que $22 \mathrm{mmHg}$ sem tratamento foi de $83 \%{ }^{(13)}$.

Em uma população de 41 pacientes (56 olhos) operados de viscocanalostomia e seguidos durante 12 meses, Drusedau et al., relata uma taxa de sucesso absoluta $(\mathrm{PIO}<21 \mathrm{mmHg}$ sem tratamento) de $36 \%$ e uma taxa de sucesso relativa $(\mathrm{PIO}<21 \mathrm{mmHg}$ com tratamento) de $79 \%$, a pressão intra-ocular média passando de $28,1 \pm 7,4 \mathrm{mmHg}$ no pré-operatório para $17,8 \pm 3,8 \mathrm{mmHg}$ no pós-operatório. As complicações pós-operatórias são limitadas a $2 \%$ de hipotonia, $17 \%$ de Seidel e $2 \%$ de catarata $^{(27)}$.

Wishart et al., praticaram a viscocanalostomia em 105 olhos com um tempo médio de acompanhamento de 36 meses (9 a 60 meses). A taxa de sucesso no final do acompanhamento, para uma PIO $<21 \mathrm{mmHg}$ sem medicação hipotensora, foi de $92,6 \%{ }^{(28)}$.

\section{Viscocanalostomia - Estudos prospectivos}

Um estudo publicado por Carassa et al., compara de maneira prospectiva a viscocanalostomia e a trabeculectomia. As pressões médias inicial e final são comparáveis nos dois grupos, respectivamente $24,6 \pm 10,6 \mathrm{mmHg}$ e $14,0 \pm 2,6 \mathrm{mmHg}$ no grupo da trabeculectomia (acompanhamento médio de 11,0 \pm 1,5 meses) e $22,3 \pm 7,4 \mathrm{mmHg}$ e 13,3 $\pm 3,8 \mathrm{mmHg}$ no grupo da viscocanalostomia (acompanhamento médio de 10,9 $\pm 1,8$ meses) ${ }^{(29)}$.

Sunaric-Mégevand, Leuenberger., em uma série de 67 olhos de 67 pacientes portadores de glaucoma de ângulo aberto, relatam $88 \%$ de sucesso global da viscocanalostomia ao final de 3 anos ( $\mathrm{PIO}<21 \mathrm{mmHg}$ com ou sem tratamento hipotensor e redução da PIO de mais de 30\%). Para uma $\mathrm{PIO}<$ $21 \mathrm{mmHg}$ sem tratamento, a taxa de sucesso é de 68\% em 1 ano e $59 \%$ em 3 anos. Nenhuma complicação importante foi observada nesta série ${ }^{(30)}$.

Jonescu-Cuypers et al., são menos otimistas em relação a viscocanalostomia, pois, em um estudo prospectivo randomizado comparando ao final de 6 meses os resultados da trabeculectomia (10 olhos) aos da viscocanalostomia (10 olhos), eles constatam que $50 \%$ dos olhos operados de trabeculectomia tem uma PIO entre 7 e $20 \mathrm{mmHg}$ sem tratamento, ao passo que nenhum dos olhos operados de viscocanalostomia respondeu a este critério de sucesso ${ }^{(31)}$.

Em uma série prospectiva e consecutiva de casos (não randomizado) de 57 olhos de 57 pacientes com glaucoma primário ou secundário de ângulo aberto sem controle clínico adequado, Shaarawy et al., realizaram a viscocanalostomia e obtiveram, ao final de 5 anos, uma taxa de sucesso de $90 \%$ para uma $\mathrm{PIO}<21 \mathrm{mmHg}$ com ou sem medicação e de $60 \%$ para uma $\mathrm{PIO}<21 \mathrm{mmHg}$ sem medicação ${ }^{(32)}$.

Recentemente três estudos prospectivos randomizados foram publicados mostrando resultados similares. No primeiro, Carassa et al., compararam a viscocanalostomia com a trabeculectomia em acompanhamento até 2 anos, mostrando que $76 \%$ dos pacientes submetidos a viscocanalostomia apresentaram PIO entre 6 e $21 \mathrm{mmHg}$ contra $80 \%$ dos pacientes submetidos a trabeculectomia. Esta diferença não sendo estatisticamente significante ${ }^{(33)}$. No segundo estudo, O'Brart et al., compararam a viscocanalostomia e a trabeculectomia associando um adjuvante antimetabólito. Neste estudo, encontrou-se uma melhor redução da PIO após 12 meses no grupo da trabeculectomia (PIO $<21 \mathrm{mmHg}$ sem medicação hipotensora 
em $91 \%$ dos casos após trabeculectomia contra $60 \%$ dos casos no grupo da viscocanalostomia) ${ }^{(34)}$. No mais recente estudo prospectivo randomizado, Yalvac et al., acompanharam pacientes submetidos a viscocanalostomia e a trabeculectomia por 3 anos, encontrando uma taxa de sucesso completa ( $\mathrm{PIO}<21 \mathrm{mmHg}$ sem medicação) em 55,1\% no grupo da trabeculectomia e $35,3 \%$ no grupo da viscocanalostomia ${ }^{(35)}$. Estes três estudos concordam em suas conclusões, quais sejam: a trabeculectomia promove uma maior redução da PIO, porém com maior número de complicações, maior desconforto e acompanhamento mais freqüente.

\section{Esclerectomia profunda não penetrante com trabeculectomia externa - Estudos retrospectivos}

Os resultados dos principais estudos retrospectivos da esclerectomia profunda não penetrante com trabeculectomia externa estão resumidos na tabela 1. Estes resultados são difíceis de comparar, visto que as técnicas cirúrgicas, os critérios de sucesso, os tipos de glaucomas operados e o tempo de acompanhamento são variáveis de um estudo para outro.

Globalmente, esses estudos mostraram que a esclerectomia profunda não penetrante parece abaixar a $\mathrm{PIO}$ em torno de $15 \mathrm{mmHg}$ e que a utilização de implantes de drenagem desenvolvidos para manter uma câmara de descompressão intraescleral (Aquaflow ${ }^{\circledR}$, Skgel $^{\circledR}$, T-Flux $^{\circledR}$ ) ou antimetabólitos podem facilitar o controle pressórico.

É igualmente difícil comparar os resultados dos estudos retrospectivos da cirurgia não penetrante aos da trabeculectomia, pois as taxas de sucesso da trabeculectomia variam segundo o critério de escolha. Por exemplo, Nouri-Mahdavi et al., relatam uma taxa de sucesso de $40 \%$ em 5 anos para uma $\mathrm{PIO}<21 \mathrm{mmHg}$ sem tratamento hipotensor. Esta taxa de sucesso aumenta para $81 \%$ se o critério de sucesso muda para uma PIO de menos de $21 \mathrm{mmhg}$ com tratamento hipotensor ${ }^{(45)}$. Migdal et al., encontraram taxas de sucesso a longo prazo elevadas após trabeculectomia. Este achado pode ser explicado pela seleção de pacientes, os quais eram submetidos à cirurgia antes de um tratamento clínico com colírios por um longo período, o que sabidamente interfere na cicatrização e na manutenção do funcionamento da fístula filtrante ${ }^{(46)}$.

Os resultados retrospectivos da esclerectomia profunda não penetrante com trabeculectomia externa relatados na tabela 1 mostram sucesso pressórico comparável ao obtido após trabeculectomia, no entanto com menor índice de complicações e melhor recuperação visual ${ }^{(47)}$.

\section{Esclerectomia profunda não penetrante com trabeculectomia externa - Estudos prospectivos}

\section{a) Não randomizados}

Ambresin et al., realizaram estudo em 20 pacientes portadores de glaucoma de ângulo aberto não controlados com medicação tópica, que já haviam sido submetidos, previamente, a uma trabeculectomia em um olho. Nestes pacientes, foi realizada uma esclerectomia profunda com implante de colágeno no outro olho. A trabeculectomia foi estudada retrospectivamente e a esclerectomia profunda, prospectivamente. O tempo de acompanhamento médio foi de 24,3 meses. Os resultados pressóricos foram comparáveis aos da trabeculectomia com menos complicações no pós-operatório precoce ${ }^{(48)}$.

Shaarawy et al., em outro estudo prospectivo não randomizado acompanharam, por um tempo médio de 64 meses, 105 olhos submetidos a esclerectomia profunda com implante de colágeno não controlados com medicação hipotensora. A PIO média pré-operatória era de $26,8 \pm 7,7 \mathrm{mmHg}$ e a PIO média pósoperatória no mês 78 foi de $12 \pm 3 \mathrm{mmHg}$. Aos 96 meses, a taxa de sucesso para uma PIO $<21$ mmHg sem medicação hipotensora foi de $57 \%{ }^{(49)}$.

\section{b) Randomizados}

El Sayyad et al., estudaram uma série de 78 olhos de 39 pacientes com acompanhamento médio de 12 meses. Nestes pacientes, foi realizada uma esclerectomia profunda em um olho e uma trabeculectomia no outro olho. No décimo segundo mês, uma PIO < $21 \mathrm{mmHg}$ foi encontrada em $93 \%$ de olhos operados de esclerectomia profunda e $94,9 \%$ de olhos submetidos a trabeculectomia ${ }^{(50)}$.

\begin{tabular}{|c|c|c|c|c|c|c|}
\hline Autores & $\mathbf{n}$ & Adjuvantes & PIO pré & PIO pós & $\mathbf{T}$ & Sucesso \\
\hline Bas, Goethals, 1999(36) & 34 & & $25,6 \pm 7,3$ & $15,3 \pm 4,3$ & 5,3 & $92,0 \%$ \\
\hline Hamard et al., $1999^{(37)}$ & $\begin{array}{l}27 \\
15\end{array}$ & $\begin{array}{l}5 \mathrm{FU} \\
\text { aquaflow }\end{array}$ & $\begin{array}{l}23,5 \pm 5,1 \\
22,6 \pm 6,9\end{array}$ & $\begin{array}{l}15,5 \pm 2,9 \\
16,2 \pm 3,9\end{array}$ & $\begin{array}{l}11,0 \\
11,0\end{array}$ & $\begin{array}{l}57,3 \% \\
66,0 \%\end{array}$ \\
\hline Massy et al., 1999(38) & 50 & & $24,3 \pm 7,2$ & $14,8 \pm 4,6$ & 14,2 & $81,0 \%$ \\
\hline Demailly et al., $1997^{(39)}$ & $\begin{array}{r}148 \\
55\end{array}$ & $\begin{array}{c}\text { aquaflow } \\
5 \mathrm{FU}\end{array}$ & $\begin{array}{l}23,3 \pm 5,2 \\
24,1 \pm 6,3\end{array}$ & $\begin{array}{l}16,1 \pm 4,7 \\
15,8 \pm 4,6\end{array}$ & $\begin{array}{l}20,0 \\
20,0\end{array}$ & $\begin{array}{l}68,0 \% \\
69,0 \%\end{array}$ \\
\hline Shaarawy et al., $2001^{(40)}$ & 105 & aquaflow & $26,8 \pm 7,0$ & $11,8 \pm 3,0$ & 43,2 & $62,0 \%$ \\
\hline Lachkar et al., 2004(41) & 258 & aquaflow & $24,5 \pm 5,9$ & $15,6 \pm 3,5$ & 60,0 & $66,5 \%$ \\
\hline Mermoud et al., 1999(42) & 44 & aquaflow & $26,7 \pm 7,3$ & $14,0 \pm 3,5$ & 14,4 & $69,0 \%$ \\
\hline Muñoz Negrete et al., $2003^{(43)}$ & 39 & & $23,1 \pm 8,8$ & $16,2 \pm 5,5$ & 12,0 & $71,9 \%$ \\
\hline Guedes, Guedes, $2005^{(44)}$ & 104 & MMC & $22,6 \pm 4,9$ & $14,2 \pm 2,9$ & 19,4 & $76,0 \%$ \\
\hline
\end{tabular}




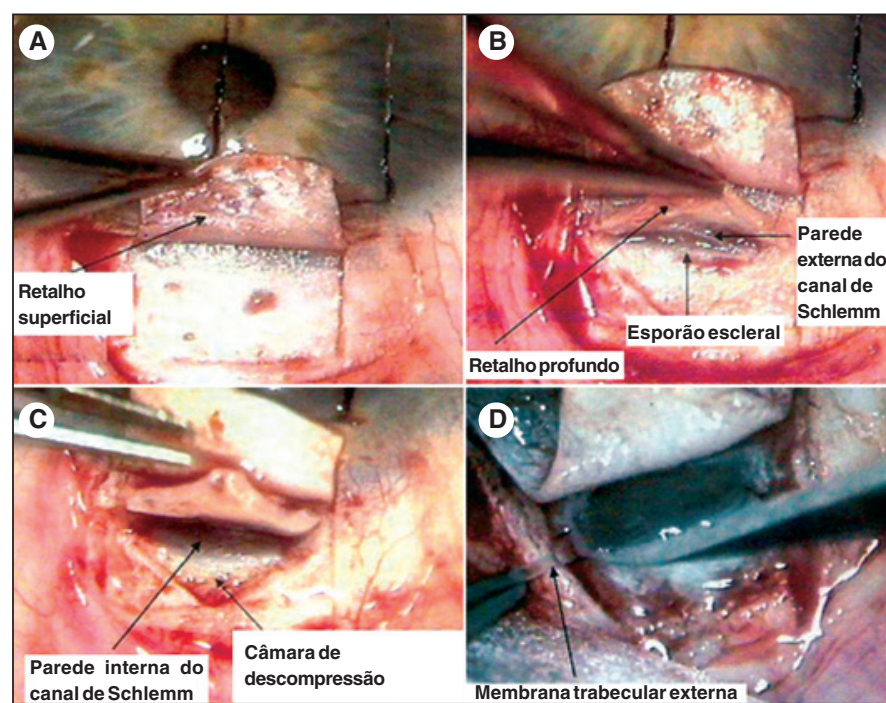

Figura 1 - A: Retalho escleral superficial; B: Retalho escleral profundo e abertura do canal de Schlemm; C: Exposição do fundo do canal de Schlemm; D: Retirada da membrana trabecular externa

Lachkar relata em uma série de 36 olhos acompanhados por 18 meses uma taxa de sucesso similar $(\mathrm{PIO}<21 \mathrm{mmHg}$ sem tratamento). Para os olhos operados de esclerectomia profunda a taxa foi de $80 \%$ e para os de trabeculectomia, $88 \%$. Após dois anos, a PIO média é equivalente nos dois grupos ${ }^{(51)}$.

Em 2002, Kozobolis et al., realizam um estudo submetendo 90 olhos a esclerectomia profunda divididos em dois grupos: o primeiro sem aplicação de adjuvantes e o segundo grupo com aplicação per-operatória de mitomicina C. Eles concluem que o uso intra-operatório de mitomicina $\mathrm{C}$ durante a esclerectomia profunda reduz a PIO pós-operatória e aumenta a taxa de sucesso do procedimento ${ }^{(52)}$.

A utilização ou não de implante de colágeno foi estudada por Shaarawy et al. Estes autores acompanharam 104 olhos, os quais foram randomizados, para receber ou não o implante. Ao final do estudo, o uso do implante aumentou a taxa de sucesso e diminuiu a necessidade de medicação hipotensora pós-operatória ${ }^{(53)}$.

Cillino et al., compararam a eficácia da esclerectomia profunda sem implante com trabeculectomia usando "punch" de Crozafon-De Laage e avaliaram o efeito de se combinar uma cirurgia de facoemulsificação ao procedimento anti-glaucomatoso. Trinta e dois olhos de 65 pacientes foram submetidos a esclerectomia profunda, sendo 17 como procedimento isolado e 15 combinados com facoemulsificação. Trinta e três olhos foram submetidos a trabeculectomia, sendo 18 isolada e 15 associada a facoemulsificação. Nenhum adjuvante foi usado, incluindo goniopuntura a laser, lise de sutura a laser ou antimetabólitos. O tempo médio de acompanhamento foi de 22,5 \pm 2,5 meses. Como conclusão, ambas as técnicas, esclerectomia profunda ou trabeculectomia isoladas, controlaram eficazmente a PIO ao final do estudo. A facoemulsificação combinada com o procedimento filtrante (penetrante ou não) não interfere no resultado final ${ }^{(54)}$.

\section{Estudos interessantes e relevantes}

Com a finalidade de se estudar a variação da PIO, assim como a presença de picos pressóricos após a cirurgia não penetrante, Guedes et al., aplicaram o teste de sobrecarga hídrica a cinco grupos de pacientes, perfazendo um total de 97 olhos. Eles estudaram 20 indivíduos normais (não portadores de glaucoma), 21 glaucomatosos não tratados, 15 glaucomatosos em uso constante de tratamento clínico hipotensor (timolol 0,5\% + dorzolamida em associação fixa), 21 glaucomatosos após uma esclerectomia profunda não penetrante e 20 glaucomatosos após uma trabeculectomia. O tempo médio de acompanhamento dos grupos após cirurgia era semelhante, em torno de um ano. A amplitude de variação da PIO, bem como o pico pressórico, após a sobrecarga hídrica foi similar no grupo normal e nos grupos da esclerectomia profunda e da trabeculectomia, confirmando os achados que o procedimento cirúrgico, seja ele penetrante ou não, é bastante eficaz em evitar picos pressóricos ${ }^{(55)}$.

Egrilmez et al., e Corcostegui et al., estudaram o astigmatismo corneano induzido pela cirurgia não penetrante. Os primeiros concluem que, apesar de uma dissecção escleral de maiores dimensões, a cirurgia não penetrante induz a menor astigmatismo do que a trabeculectomia. Fato este explicado pela sutura do retalho escleral, que na trabeculectomia, às vezes tem que ser mais apertada ${ }^{(56)}$. O segundo grupo avaliou a influência da esclerectomia profunda na indução de astigmatismo em cirurgias combinadas, concluindo que não há significância clínica desta mudança refrativa ${ }^{(57)}$.

Shaarawy et al., avaliaram a PIO no primeiro dia de pósoperatório como um fator de prognóstico positivo para a cirurgia. A relação entre a PIO no primeiro dia de pós-operatório e a subseqüente necessidade de medicação hipotensora e realização de goniopuntura com Nd:YAG laser foi estudada. O tempo médio de acompanhamento foi de 43,2 $\pm 14,3$ meses. Os olhos com $\mathrm{PIO}=5 \mathrm{mmHg}$ apresentaram uma performance $\mathrm{e}$ uma sobrevida, medida por uma estratificação variável de Kaplan-Meier, bem superior àqueles com PIO > $5 \mathrm{mmHg}$ no primeiro dia após uma escleretomia profunda. Concluem que a PIO no primeiro dia de pós-operatório pode ser considerada como um fator positivo de prognóstico para a esclerectomia profunda ${ }^{(58)}$.

Um estudo de meta-análise da cirurgia não penetrante foi realizado por Cheng et al. Trinta e sete artigos foram incluídos na avaliação. A taxa de sucesso cumulativa para $\mathrm{PIO}<21 \mathrm{mmHg}$ sem tratamento hipotensor foi de $67,8 \%$. Os autores concluem que a cirurgia não penetrante do trabeculado é a melhor terapia disponível para glaucomas de ângulo aberto não controlados clinicamente $^{(59)}$.

\section{DISCUSSÃO}

O conceito de se reduzir a pressão intra-ocular (PIO) por meio de uma cirurgia que preserve o trabeculado mais interno tem recentemente atraído muito interesse. Um número grande 
de publicações tem aparecido em periódicos respeitados. A parte central desta técnica, qualquer que seja sua variante, é a preservação de uma câmara anterior intacta, o que dispensa uma iridectomia e evita as conseqüências da hipotonia no pós-operatório imediato, minimizando a freqüência de hifema, descolamento de coróide, câmara anterior rasa e catarata ${ }^{(4,60)}$.

Atualmente, discute-se muito o papel da cirurgia não penetrante como sucessora da trabeculectomia, atual padrão-ouro no tratamento cirúrgico do glaucoma de ângulo aberto. Intervenções cirúrgicas eficazes no glaucoma devem ser capazes de controlar a PIO em longo prazo, acompanhando a cronicidade da doença. Já está bem estabelecido que, em médio prazo, a cirurgia não penetrante apresenta resultados tensionais similares aos da trabeculectomia, com uma incidência bem menor de complicações pós-operatórias. Resultados em longo prazo têm começado a aparecer, inclusive com estudos prospectivos randomizados, confirmando o achado anterior ${ }^{(60)}$.

Outro ponto bastante discutido se refere à técnica cirúrgica em si. Alguns relatam que a sua realização é difícil e envolve riscos no seu aprendizado ${ }^{(60)}$. No entanto, existem muitas evidências sobre a segurança deste aprendizado e a conversão de uma técnica à outra. Sanchez et al., compararam os resultados da trabeculectomia clássica com a esclerectomia profunda transformada em trabeculectomia, a fim de se estudar o risco no aprendizado desta nova técnica cirúrgica. Eles chegaram à conclusão que uma esclerectomia profunda complicada por uma perfuração da membrana trabecular residual tem a mesma taxa de sucesso de uma trabeculectomia clássica em longo prazo $^{(61)}$. Drolsum avaliou prospectivamente seus primeiros 44 casos de esclerectomia profunda a fim de observar a segurança da conversão da trabeculectomia. Perfuração acidental na dissecção ocorreu em $8,3 \%$ e ao final de um ano a taxa de sucesso foi de $61,4 \%$ (PIO < $21 \mathrm{mmHg}$ sem medicação) com nenhum relato de hipotonia pós-operatória ${ }^{(62)}$. A conversão de uma técnica à outra, porém, exige um cirurgião experimentado e habituado com a dissecção escleral. A fim de se obter sucesso com a cirurgia não penetrante, a dissecção deve ser feita corretamente. Isto inclui a exérese do retalho escleral profundo e a retirada da membrana trabecular externa, a qual engloba a parede interna do canal de Schlemm e o trabeculado juxtacanalicular.

A goniopuntura a laser pode ser realizada em qualquer estágio do pós-operatório, quando a PIO se eleva a níveis acima daqueles definidos como valores de PIO-alvo. A goniopuntura consiste em se abrir, por meio de aplicação de Nd:YAG laser, a fina membrana trabecular residual no sítio operatório. A necessidade desta aplicação diminui à medida que a dissecção escleral e a retirada da membrana trabecular externa são feitas corretamente. Esta medida não deve ser considerada como critério de insucesso cirúrgico, mas sim como mais uma alternativa disponível para o sucesso do procedimento. Se não correríamos o risco de considerar a lise de sutura da trabeculectomia e capsulotomia a laser da cirurgia de catarata também como critérios de insucesso do procedimento respectivo.
No que diz respeito ao custo da cirurgia, se levarmos em conta o preço dos implantes disponíveis, a cirurgia não penetrante passa a ter a desvantagem de ser mais dispendiosa do que a clássica trabeculectomia. No entanto, alguns autores advogam a possibilidade, com bons resultados, da realização da cirurgia não penetrante sem utilização de implantes ${ }^{(37-38,44,52)}$. Nestes casos, é indispensável o uso de antimetabólitos, largamente usados pelos cirurgiões habituados com a trabeculectomia. Deste modo, o custo das duas técnicas se revela semelhante.

A qualidade de vida proporcionada pela cirurgia não penetrante ao paciente e ao médico é superior àquela proporcionada pela trabeculectomia. Os pacientes têm um pós-operatório sem baixa de acuidade visual, sem risco de hipotonia, podendo manter suas atividades diárias com um mínimo de restrições. Já os médicos se beneficiam muito, fazendo um acompanhamento pós-operatório mais tranqüilo com menos visitas, o que inclusive diminui o custo global da cirurgia.

Existe, no entanto, ainda uma necessidade premente de estudos prospectivos e randomizados bem conduzidos e em escala mundial a fim de se comparar a cirurgia não penetrante, ou qualquer outra técnica cirúrgica, com a trabeculectomia. Apesar disto, este "novo" procedimento encontra-se já bem estabelecido como mais uma alternativa cirúrgica no tratamento do glaucoma de ângulo aberto.

\section{CONCLUSÃO}

A literatura levantada mostra que a cirurgia filtrante não penetrante permite controlar a pressão intra-ocular dos pacientes portadores de glaucoma de ângulo aberto minimizando os riscos de hipotonia. Os resultados pressóricos obtidos são comparáveis aos da trabeculectomia com menos incidência de complicações pós-operatórias precoces.

O controle pressórico é melhor quando a esclerectomia está associada a uma trabeculectomia externa. Outros estudos prospectivos serão necessários para comprovar estes resultados e avaliar o interesse dos diferentes tipos de implante no decorrer da cirurgia.

\section{ABSTRACT}

Recently, many discussions occurred concerning non-penetrating filtering surgery. Viscocanalostomy and deep sclerectomy with external trabeculectomy are the most practiced techniques. Their goal is to reduce intraocular pressure by enhancing the natural aqueous outflow, while reducing its resistance. Both techniques involve the removal of a deep scleral flap, the external wall of Schlemm's canal and corneal stroma behind the anterior trabecular meshwork and Descemet membrane, thus creating a scleral lake, where the aqueous humor remains until it is absorbed in many different ways. In viscocanalostomy, a high-molecular viscoelastic substance is injected into the ostia of Schlemm's canal in order to enlarge it 
and its collectors channels. In deep sclerectomy with external trabeculectomy, the inner wall of Schlemm's canal is removed with adjacent trabecular layers. Studies, both retrospective and prospective, demonstrated similar tensional results when compared to classical trabeculectomy, with fewer complications and better visual recovery after non-penetrating procedure. The aim of this study is to expose the non-penetrating surgery techniques, mechanism of action and results based on the analysis of published literature.

Keywords: Glaucoma/sugery; Filtering surgery; Intraocular pressure/physiology; Postoperative care; Visual acuity; Aqueous humor/secretion; Sclerostomy

\section{REFERÊNCIAS}

1. Shields MB. An overview of glaucoma. In: Shields MB. Textbook of glaucoma. $4^{\text {th }}$ ed. Baltimore: Williams and Wilkins; 1998. p.1-2.

2. Béchetoile A. Le glaucome primitif à angle ouvert. In : Béchetoile A. Les glaucomes. Angers: Japperenard; 1997. p.324-8. v.2.

3. Baudouin C. [When should glaucoma be surgically treated ?] J Fr Ophtalmol. 2001;24(10):1103-9. French.

4. Hamard P, Lachkar Y. [Non penetrating filtering sugery, evolution and results]. J Fr Ophtalmol. 2002;25(5):527-36. French.

5. Seiler T, Wollensak J. The resistance of the trabecular meshwork to aqueous humor outflow. Graefes Arch Clin Exp Ophthalmol. 1985;223(2):88-91.

6. Rosenquist R, Epstein D, Melamed S, Johnson M, Grant WM. Outflow resistance of enucleated human eyes at two different perfusion pressures and different extents of trabeculotomy. Curr Eye Res. 1989;8(12):1233-40.

7. Nesterov AP. Role of blockade of Schlemm's canal in pathogenesis of primary open-angle glaucoma. Am J Ophthalmol. 1970;70(5):691-6.

8. Krasnov MM. Externalization of Schlemm's canal (sinusotomy) in glaucoma. Br J Ophthalmol. 1968;52(2):157-61.

9. Zimmermann TJ, Kooner KS, Ford VJ, Olander KW, Mandlekorn RM, Rawlings FE, et al. Effectiveness of non-penetrating trabeculectomy in aphakic patients with glaucoma. Ophthalmic Surg. 1984;15(1):44-50.

10. Zimmermann TJ, Kooner KS, Ford VJ, Olander KW, Mandlekorn RM, Rawlings EF, et al. Trabeculectomy versus non-penetrating trabeculectomy: a retrospective study of two procedures in phakic patients with glaucoma. Ophthalmic Surg. 1984;15(9):734-40.

11. Koslov VI, Bagrov SN, Anisimova SY. Non-penetrating deep sclerectomy with collagen implant. IRTC Eye Microsurgery. 1990:3:44-6. Moscow: RSFSR Ministry of Public Health.

12. Valtot F. La chirurgie non perforante du glaucome: les différentes technique et leurs modes d'action. In: Béchetoille A. editor. $7^{\circ}$ Symposium Recherche et Glaucome; 1999; Orlando, 24 Octobre. Paris: EDK; 2000. p.75-84.

13. Stegmann R, Pienaar A, Miller D. Viscocanalostomy for open-angle glaucoma in black African patients. J Cataract Refract Surg. 1999;25(3):316-22.

14. Arenas-Archilla E. Ab externo trabeculectomy [letter]. Highlights Ophthalmol. (Édition Française). 1991;19:60-6.

15. Demailly P, Jeanteur-Lunel MN, Berkani M, Ecoffet M, Kopel J, Kretz G, et al. [Non-penetrating deep sclerectomy combined with a collagen implant in primary open-angle glaucoma. Medium-term retrospective results]. J Fr Ophtalmol. 1996;19(11):659-66.

16. Koslov VI, Bagrov SN, Anisimova SY. Non-penetrating deep sclerectomy with collagen implant. IRTC Eye Microsurgery. 1990:3:44-6. Moscow: RSFSR Ministry of Public Health.

17. Guedes VMP, Guedes RAP. Esclerectomia profunda não penetrante: resultados em médio prazo dos primeiros pacientes operados. Rev Bras Oftalmol. 2001;60(1):20-4.

18. Smit BA, Johnstone MA. Effects of viscocanalostomy on the histology of Schlemm's canal in primate eyes [abstract]. Invest Ophthalmol Vis Sci. 2000; 41(4):S578.

19. Mermoud A. [Deep sclerectomy: surgical technique] J Fr Ophtalmol. 1999; 22(7):781-6. French.
20. Bresson-Dumont H, Bray JM de, Bechetoille A. [Color doppler ultrasonography of short para-optic ciliary arteries in vascular glaucoma]. J Fr Ophthalmol. 1999;22(7):743-8. French.

21. Hamard P, Sourdille P, Valtot F, Baudouin C. [Evaluation of confocal microscopy in the analysis of the external trabecular membrane during deep nonpenetrating sclerectomy]. J Fr Ophtalmol. 2001;24(1):29-35. French.

22. Marchini G, Marraffa M, Brunelli C, Morbio R, Bonomi L. Ultrasound biomicroscopy and intraocular-pressure-lowering mechanisms of deep sclerectomy with reticulated hyaluronic acid implant. J Cataract Refract Surg. 2001;27(4): 507-17.

23. Delarive T, Rossier A, Rossier S, Ravinet E, Shaarawy T, Mermoud A. Aqueous dynamic and histological findings after deep sclerectomy with collagen implant in an animal model. Br J Ophthalmol 2003;87(11):1340-4. Comment in: Br J Ophthalmol. 2003;87(11):1310.

24. Bensaid A, Lachkar Y. Sclérectomie profonde avec trabéculectomie externe non perforante: indications, complications, surveillance. Réflexions Ophtalmologique. 2003;63(8):15-6.

25. Sourdille P, Santiago PY, Villain F, Yamamichi M, Tahi H, Parel JM, et al. Reticulated hyaluronic acid implant in non perforating trabecular surgery. J Cataract Refract Surg. 1999;25(3):332-9. Comment in: J Cataract Refract Surg. 1999;25(3):298-300; J Cataract Refract Surg. 1999;25(10):1309.

26. Carassa R, Bettin P, Fiori M, Brancato R. Viscocanalostomy: a pilot study. Eur J Ophthalmol. 1998;8(2):57-61.

27. Drusedau MU, Von Wolff K, Bull H, Von Barsewisch D. Viscocanalostomy for primary open-angle glaucoma: the Gross Pankow experience. J Cataract Refract Surg. 2000;26(9):1367-73.

28. Wishart PK, Wishart MS, Porooshani H. Viscocanalostomy and deep sclerectomy for the surgical treatment of glaucoma: a longterm follow-up. Acta Ophthalmol Scand. 2003;81(4):343-8.

29. Carassa RG, Betin O, Fiori M. Viscocanalostomy versus trabeculectomy: a 12 month prospective trial [abstract]. Invest Ophthalmol Vis Sci. 2000;41(4):S744.

30. Sunaric-Mégevand G, Leuenberger P. Results of viscocanalostomy for primary open-angle glaucoma. Am J Ophthalmol. 2001;132(2):221-8.

31. Jonescu-Cuypers C, Jacobi P, Konen W, Krieglstein G. Primary viscocanalostomy versus trabeculectomy in white patients with open-angle glaucoma: a randomized clinical trial. Ophthalmology. 2001;108(2):254-8. Comment in: Ophthalmology. 2002;109(3):410-1; Ophthalmology. 2002;109(3):411-2; Ophthalmology. 2003;110(11):2259-60.

32. Shaarawy T, Nguyen C, Schnyder C, Mermoud A. Five year results of viscocanalostomy. Br J Ophthalmol. 2003;87(4):441-5.

33. Carassa RG, Bettin P, Fiori M, Brancato R. Viscocanalostomy versus trabeculectomy in white adults affected by open-angle glaucoma: a 2-year randomized, controlled trial. Ophthalmology. 2003;110(5):882-7.

34. O'Brart DP, Shiew M, Edmunds B. A randomised, prospective study comparing trabeculectomy with viscocanalostomy with adjunctive antimetabolite usage for the management of open angle glaucoma uncontrolled by medical therapy. Br J Ophthalmol. 2004;88(8):1012-7.

35. Yalvac IS, Sahin M, Eksioglu U, Midillioglu IK, Aslan BS, Duman S. Primary viscocanalostomy versus trabeculectomy for primary open-angle glaucoma: three-year prospective randomized clinical trial. J Cataract Refract Surg. 2004;30(10):2050-7.

36. Bas JM, Goethals MJH. Non-penetrating deep sclerectomy preliminary results. Bull Soc Belge Ophthalmol. 1999;272:55-9.

37. Hamard P, Plaza L, Kopel J, Quesnot S, Hamard H. [Deep nonpenetrating sclerectomy and open angle glaucoma. Intermediate results form the first operated patients]. J Fr Ophtalmol. 1999;22(1):25-31. French.

38. Massy J, Gruber D, Muraine M, Brasseur G. [Non-penetrating deep sclerectomy in the surgical treatment of chronic open-angle glaucoma. Mid-term results]. J Fr Ophtalmol. 1999;22(3):292-8. French.

39. Demailly P, Lavat P, Kretz G, Jeanteur-Lunel MN. Non-penetrating deep sclerectomy (NPDS) with or without collagen device (CD) in primary openangle glaucoma: middle-term retrospective study. Int Ophthalmol. 1997;20 (1-3):131-40

40. Shaarawy T, Karlen M, Sanchez E, Achache F, Schnyder C, Mermoud A. Five year results of deep sclerectomy with collagen implant. J Cataract Refract Surg. 2001;27(11):1770-8.

41. Lachkar Y, Neverauskiene J, Jeanteur-Lunel MN, Gracies H, Berkani M, Ecoffet M, et al. Nonpenetrating deep sclerectomy: a 6-year retrospective study. Eur J Ophthalmol. 2004;14(1):26-36.

42. Mermoud A, Schnyder CC, Sickenberg M, Chiou AG, Hediguer SE, Faggioni R. Comparison of deep sclerectomy with collagen implant and trabeculectomy in open angle glaucoma. J Cataract Refract Surg. 1999;25(3):323-31. Comment in: J Cataract Refract Surg. 1999;25(3):297; J Cataract Refract Surg. 1999; 25(3):298-300; J Cataract Refract Surg. 1999;25(10):1309.

43. Muñoz Negrete FJ, Rebolleda G, Noval S. [Non penetrating deep sclerectomy 
combined with phacoemulsification. Results and complications]. Arch Soc Esp Oftalmol. 2003;78(9):499-506.

44. Guedes RA, Guedes VM. [Nonpenetrating deep sclerectomy in Brazil: 3-year retrospective study]. J Fr Ophtalmol. 2005;28(2):191-6. French.

45. Nouri-Mahdavi K, Brigatti L, Weitzman M, Caprioli J. Outcomes of trabeculectomy for primary open-angle glaucoma. Ophthalmology. 1995;102(12):1760-9.

46. Migdal CS, Gregory W, Hitchings R. Long-term functional outcome after early surgery compared with laser and medicine in open-angle glaucoma. Ophthalmology. 1994;101(10):1651-6; discussion 1657.

47. Vass C, Menapace R. Surgical strategies in patients with combined cataract and glaucoma. Curr Opin Ophthalmol. 2004;15(1):61-6.

48. Ambresin A, Shaarawy T, Mermoud A. Deep sclerectomy with collagen implant in one eye compared with trabeculectomy in the other eye of the same patient. J Glaucoma. 2002;11(3):214-20.

49. Shaarawy T, Mansouri K, Schnyder C, Ravinet E, Achache F, Mermoud A. Long-term results of deep sclerectomy with collagen implant. J Cataract Refract Surg. 2004;30(6):1225-31. Comment in: J Cataract Refract Surg. 2005;31(5): 868-9.

50. El Sayyad F, Helal M, El-Kholify M, El-Maghraby A. Nonpenetrating deep sclerectomy versus trabeculectomy in bilateral open angle glaucoma. Ophthalmology. 2000;107(9):1671-4. Comment in: Ophthalmology. 2001;108(10):1718.

51. Lachkar Y. Non penetrating deep sclerectomy with external trabeculectomy vs trabeculectomy [abstract]. Invest Ophthalmol Vis Sci. 2001;42(1):S67.

52. Kozobolis VP, Christodoulakis EV, Tzanakis N, Zacharopoulos I, Pallikaris IG. Primary deep sclerectomy versus primary deep sclerectomy with the use of mitomycin C in primary open-angle glaucoma. J Glaucoma. 2002;11(4):287-93.

53. Shaarawy T, Nguyen C, Schnyder C, Mermoud A. Comparative study bet- ween deep sclerectomy with and without collagen implant: long term follow up. Br J Ophthalmol. 2004;88(1):95-8.

54. Cillino S, Pace FD, Casuccio A, Calvaruso L, Morreale D, Vadala M, et al. Deep sclerectomy versus punch trabeculectomy with or without phacoemulsification: a randomized clinical trial. J Glaucoma. 2004;13(6):500-6.

55. Guedes RA, Guedes VM, Chaoubah A. Application du teste de surcharge hydrique après la sclérectomie profonde non perforante. J Fr Ophtalmol. 2005;28(10):1076-80.

56. Egrilmez S, Ates H, Nalcaci S, Andac K, Yagci A. Surgically induced corneal refractive change following glaucoma surgery: nonpenetrating trabecular surgeries versus trabeculectomy. J Cataract Refract Surg. 2004;30(6):1232-9.

57. Corcostegui J, Rebolleda G, Muñoz-Negrete FJ. Refractive changes after phacoemulsification combined with deep sclerectomy assisted by corneal topography. J Cataract Refract Surg. 2004;30(11):2391-6.

58. Shaarawy T, Flammer J, Smits G, Mermoud A. Low first postoperative day intraocular pressure as a positive prognostic indicator in deep sclerectomy. $\mathrm{Br}$ J Ophthalmol. 2004;88(5):658-61.

59. Cheng JW, Ma XY, Wei RL. Efficacy of non-penetrating trabecular surgery for open angle glaucoma: a meta-analysis. Chin Med J (Engl). 2004;117(7):1006-10.

60. Tan JC, Hitchings RA. Non-penetrating glaucoma surgery: the state of play. $\mathrm{Br}$ J Ophthalmol. 2001;85(2):234-7. Comment in: Br J Ophthalmol. 2002;86(12): 1460-1.

61. Sanchez E, Schnyder CC, Mermoud A. [Comparative results of deep sclerectomy transformed to trabeculectomy and classical trabeculectomy]. Klin Monatsbl Augenheilkd. 1997;210(5):261-4. French.

62. Drolsum L. Conversion from trabeculectomy to deep sclerectomy. Prospective study of the first 44 cases. J Cataract Refract Surg. 2003;29(7):1378-84.

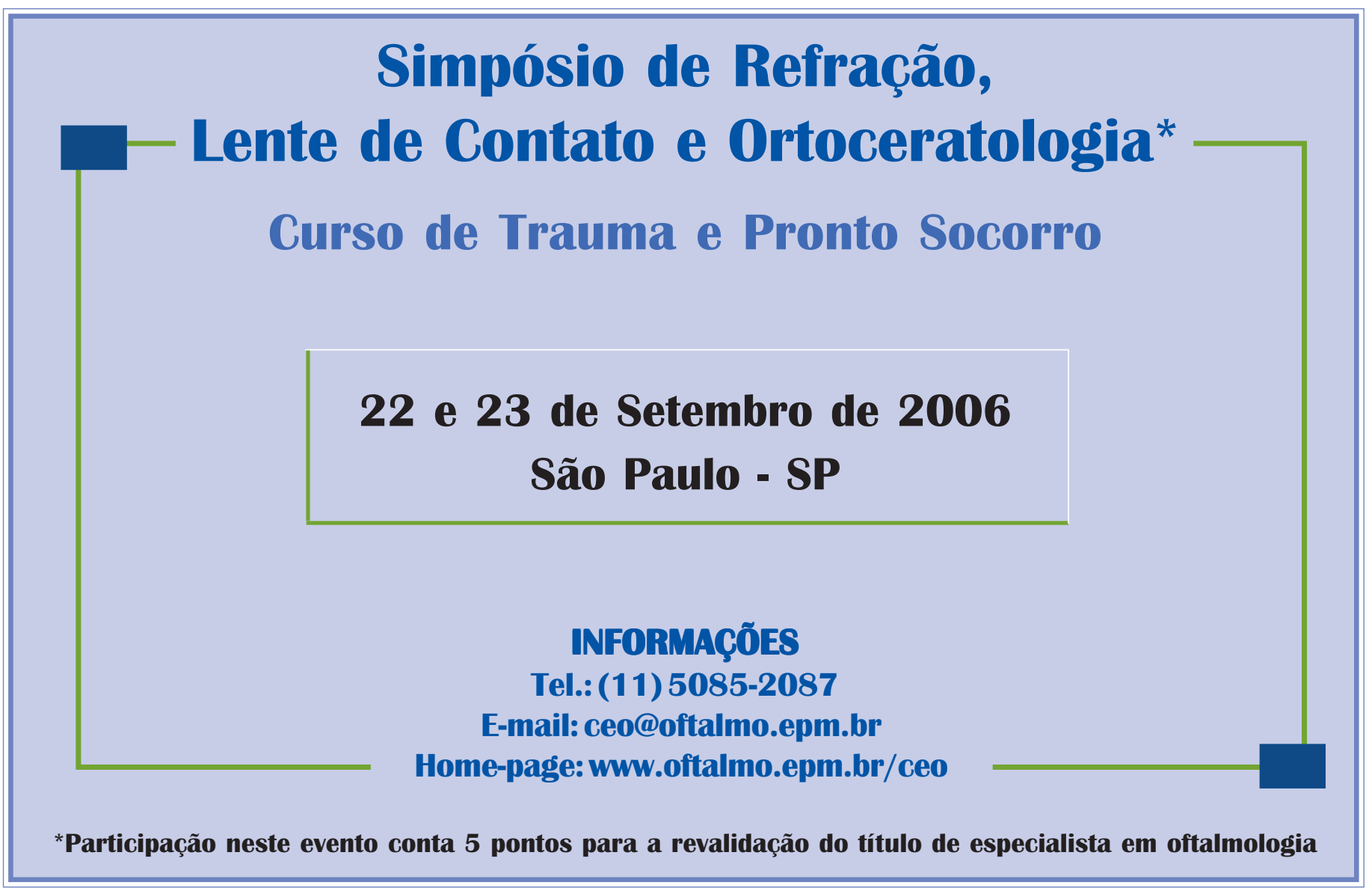

